\title{
Tension-compression asymmetry modelling: strategies for anisotropy parameters identification
}

\author{
Pedro Barros ${ }^{1, a}$, José Luís Alves ${ }^{2}$, Marta Oliveira ${ }^{1}$ and Luís Filipe Menezes ${ }^{1}$ \\ ${ }^{1}$ CEMUC, Department of Mechanical Engineering, University of Coimbra, \\ Pólo II, Rua Luís Reis Santos, Pinhal de Marrocos, 3030-788 Coimbra, Portugal \\ ${ }^{2}$ CMEMS, Microelectromechanical Systems Research Unit, University of Minho, \\ Campus de Azurém, 4800-058 Guimarães, Portugal
}

\begin{abstract}
This work presents details concerning the strategies and algorithms adopted in the fully implicit FE solver DD3IMP to model the orthotropic behavior of metallic sheets and the procedure for anisotropy parameters identification. The work is focused on the yield criterion developed by Cazacu, Plunkett and Barlat, 2006 [1], which accounts for both tension-compression asymmetry and orthotropic plastic behavior. The anisotropy parameters for a 2090-T3 aluminum alloy are identified accounting, or not, for the tension-compression asymmetry. The numerical simulation of a cup drawing is performed for this material, highlighting the importance of considering tension-compression asymmetry in the prediction of the earing profile, for materials with cubic structure, even if this phenomenon is relatively small.
\end{abstract}

\section{Introduction}

Plastic forming of metals with hexagonal closed packed (HCP) structure is still challenging due to their low ductility at room temperature and their unusual deformation characteristics, related with very pronounced anisotropy with strong asymmetry between tensile and compressive behavior. This tension-compression asymmetry is present because, unlike cubic metals (both face centered (FCC) and body centered (BCC)), hexagonal metals deform due to the activation of mechanical twinning or non-Schimd type slip at single crystal level. In contrast to slip, twinning is a directional shear mechanism: in general, shear in one direction can produce twinning while shear in the opposite direction cannot. Although less pronounced, the strength differential (SD) effect is also present in materials with cubic structure. However, this effect has been typically neglected, with the yield surface assumed to show a point-symmetry, with respect to the center, such that a stress state and its reverse state have the same flow stress absolute value [2-5].

For the correct modelling of metallic sheets mechanical behavior, it is necessary to perform experimental characterization tests. The most usual is the uniaxial tensile test, allowing to have the yield stresses and anisotropy coefficients in several directions of the sheets plane (generally, $0,15,30,45,60,75$ and 90 degrees from the rolling direction). In order to improve the description of the yield surface, it is also recommended to determine experimentally the biaxial yield stress and the biaxial anisotropy coefficient [6-8]. However, in order to characterize the metallic sheets mechanical behavior for

\footnotetext{
${ }^{\mathrm{a}}$ Corresponding author: pedro.barros@dem.uc.pt
}

compression stress states, it is necessary to avoid buckling effects. This requires the use of smaller specimens and, consequently, leading to supplementary difficulties in the acquisition and analysis of the experimental results, particularly for high strain values (see e.g. [6,9-11]).

This work is focused on evaluating the influence of taking into account tension-compression asymmetry in the numerical simulation of a cup drawing. Some details concerning the non-quadratic yield criterion proposed by Cazacu, Plunkett and Barlat (2006) are presented in the first section. Following, a description of the anisotropy parameters identification is shown for the description of the mechanical behavior of a 2090-T3 aluminum alloy. The simulation of a cup drawing is performed in order to evaluate the influence of taking into account, or not, tension-compression asymmetry in the prediction of the earing profile.

\section{Constitutive model}

The description of both anisotropic behavior and tension-compression asymmetry is possible using the model proposed by Cazacu, Plunkett and Barlat (2006) [1] The equivalent stress $\bar{\sigma}$ associated with the orthotropic form of the CPB06 yield criterion is defined as

$$
\bar{\sigma}=B\left[\left(\left|s_{1}\right|-k s_{1}\right)^{a}+\left(\left|s_{2}\right|-k s_{2}\right)^{a}+\left(\left|s_{3}\right|-k s_{3}\right)^{a}\right]^{\frac{1}{a}},
$$

where $a$ and $k$ are material parameters and $s_{1}, s_{2}$ and $s_{3}$ are the principal values of $\mathbf{s}=\mathbf{C} \boldsymbol{\sigma}^{\prime}$, determined following Barlat et al. (1997) [12]. $\sigma^{\prime}$ is the deviatoric stress tensor 
and $\mathbf{C}$ is a constant $4^{\text {th }}$-order tensor which, for 3-D stress conditions involves 9 independent anisotropy coefficients, given by

$$
\mathbf{C}=\left[\begin{array}{cccccc}
C_{11} & C_{12} & C_{13} & 0 & 0 & 0 \\
C_{12} & C_{22} & C_{23} & 0 & 0 & 0 \\
C_{13} & C_{23} & C_{33} & 0 & 0 & 0 \\
0 & 0 & 0 & C_{44} & 0 & 0 \\
0 & 0 & 0 & 0 & C_{55} & 0 \\
0 & 0 & 0 & 0 & 0 & C_{66}
\end{array}\right] .
$$

Considering isotropic conditions ( $\mathrm{C}$ equal to the $4^{\text {th }}$ order identity tensor), the coefficient $k$ alone allows for the description of the SD effect, giving a direct measure of the ratio between tensile and compressive yield stresses, as

$$
k=\frac{1-h}{1+h} \text {, with } h=\left[\frac{2^{a}-2\left(\frac{\sigma^{\mathrm{T}}}{\sigma^{\mathrm{C}}}\right)^{a}}{\left(2 \frac{\sigma^{\mathrm{T}}}{\sigma^{\mathrm{C}}}\right)^{a}-2}\right]^{1 / a} .
$$

This means that the $\sigma^{\mathrm{T}} / \sigma^{\mathrm{C}}$ ratio, i.e. the ratio between the yield stress in uniaxial tension and the yield stress in uniaxial compression, for the three principal axes is the same. However, for an anisotropic material it is not possible to define a single $\sigma^{\mathrm{T}} / \sigma^{\mathrm{C}}$ ratio and, consequently, the physical meaning of the $k$ parameter is lost [13]. $B$ is a constant (see Eq. (1)) defined such that $\bar{\sigma}$ reduces to the tensile yield stress in the rolling direction and is given by

$$
B=\left[\frac{1}{\left(\left|\phi_{1}\right|-k \phi_{1}\right)^{a}+\left(\left|\phi_{2}\right|-k \phi_{2}\right)^{a}+\left(\left|\phi_{3}\right|-k \phi_{3}\right)^{a}}\right]^{\frac{1}{a}},
$$

with

$$
\left\{\begin{array}{l}
\phi_{1} \\
\phi_{2} \\
\phi_{3}
\end{array}\right\}=\left\{\begin{array}{l}
(2 / 3) C_{11}-(1 / 3) C_{12}-(1 / 3) C_{13} \\
(2 / 3) C_{21}-(1 / 3) C_{22}-(1 / 3) C_{23} \\
(2 / 3) C_{31}-(1 / 3) C_{32}-(1 / 3) C_{33}
\end{array}\right\} .
$$

For $a=2, k=0$ and $\mathbf{C}$ equal to the $4^{\text {th }}$-order identity tensor, the von Mises yield criterion is recovered. Convexity is guaranteed for any integer $a \geq 2$ and $k \in[-1,1]$ [1]. In this work the analysis performed considers $a=2$.

In order to highlight the influence of taking, or not, into account tension-compression asymmetry, the yield criterion proposed by Barlat et al. (1991), labeled YLD91, is also used in this work. The equivalent stress $\bar{\sigma}$ associated with the orthotropic form of the YLD91 yield criterion is given as

$$
\bar{\sigma}=\left\{\frac{1}{2}\left[\left|s_{1}-s_{2}\right|^{m}+\left|s_{2}-s_{3}\right|^{m}+\left|s_{1}-s_{3}\right|^{m}\right]\right\}^{\frac{1}{m}},
$$

where $s_{1}, s_{2}, s_{3}$ are the principal values of $\mathbf{s}=\mathbf{L} \boldsymbol{\sigma}^{\prime}$ and $m$ is the exponent that controls the shape of the isotropic yield surface, usually taken as 6 or 8 for body centered (BCC) and face centered (FCC) materials, respectively [14]. $\boldsymbol{\sigma}^{\prime}$ is the deviatoric stress tensor and $\mathbf{L}$ is a constant $4^{\text {th }}$-order tensor given by

$$
\mathbf{L}=\left[\begin{array}{cccccc}
\left(c_{2}+c_{3}\right) / 3 & -c_{3} / 3 & -c_{2} / 3 & 0 & 0 & 0 \\
-c_{3} / 3 & \left(c_{3}+c_{1}\right) / 3 & -c_{1} / 3 & 0 & 0 & 0 \\
-c_{2} / 3 & -c_{1} / 3 & \left(c_{1}+c_{2}\right) / 3 & 0 & 0 & 0 \\
0 & 0 & 0 & c_{4} & 0 & 0 \\
0 & 0 & 0 & 0 & c_{5} & 0 \\
0 & 0 & 0 & 0 & 0 & c_{6}
\end{array}\right],
$$

which, for a 3-D stress state, involves only 6 independent anisotropy coefficients. Therefore, although $\mathbf{C}$ and $\mathbf{L}$ are defined using the same formalism of the linear transformation, proposed by Barlat et al. (1991) [12], they lead to different sets of anisotropic parameters.

The yield condition is defined as

$$
F(\bar{\sigma}, Y)=\bar{\sigma}-Y=0,
$$

where $Y$ is the flow stress, which depends of the hardening law selected, such that the yield stress is $Y_{0}$. The adopted constitutive model considers an associated flow rule, meaning that the yield function serves as the plastic potential for determining the plastic strain increment. The plastic strain rate tensor, $\mathbf{D}^{\mathrm{p}}$, is determined according to

$$
\mathbf{D}^{\mathrm{p}}=\dot{\lambda} \frac{\partial F(\bar{\sigma}, Y)}{\partial \boldsymbol{\sigma}},
$$

The equivalent stress is given by Eq. (1) or (6), depending on the yield criterion adopted, and $\dot{\lambda}$ is a scalar value designated by plastic multiplier that can be demonstrated to be equal to the equivalent plastic strain rate, $\dot{\bar{\varepsilon}}^{\mathrm{p}}$. The equivalent plastic strain is defined as

$$
\bar{\varepsilon}^{\mathrm{p}}=\int_{0}^{t} \dot{\bar{\varepsilon}}^{\mathrm{p}} \mathrm{d} t=\int_{0}^{t} \frac{\boldsymbol{\sigma}^{\prime}}{\bar{\sigma}}: \mathbf{D}^{\mathrm{p}} \mathrm{d} t .
$$

\section{Material parameters identification}

The anisotropy coefficients should be determined such that the yield criterion reproduces the material's mechanical behavior as close as possible. The procedure adopted is based on the minimization of an error function, evaluating the difference between the estimated values and the experimental ones, as follows 


$$
\begin{aligned}
F(\mathbf{A}) & =\sum_{\theta=0}^{90} w_{\sigma_{\theta}^{T}}\left(\sigma_{\theta}^{Y_{T}}(\mathbf{A}) / \sigma_{\theta}^{Y_{T}}-1\right)^{2} \\
& +\sum_{\theta=0}^{90} w_{\sigma_{\theta}^{C}}\left(\sigma_{\theta}^{Y_{C}}(\mathbf{A}) / \sigma_{\theta}^{Y_{C}}-1\right)^{2} \\
& +\sum_{\theta=0}^{90} w_{r_{\theta}}\left(r_{\theta}(\mathbf{A}) / r_{\theta}-1\right)^{2}, \\
& +w_{\sigma_{b}}\left(\sigma_{b}(\mathbf{A}) / \sigma_{b}-1\right)^{2} \\
& +w_{r_{b}}\left(r_{b}(\mathbf{A}) / r_{b}-1\right)^{2}
\end{aligned}
$$

where $\mathbf{A}$ represents the set of yield parameters. $\sigma_{\theta}^{Y_{T}}, \sigma_{\theta}^{Y_{C}}$ and $r_{\theta}$ are the experimental yield stresses in tension, compression and $r$-values, respectively, obtained from the uniaxial tensile and compression tests for a specific orientation $(\theta)$ to RD. $\sigma_{b}$ is the experimental yield stress obtained from the equibiaxial test and $r_{b}$ can be obtained from the disc compression test. $\sigma_{\theta}^{Y_{T}}(\mathbf{A}), \sigma_{\theta}^{Y_{C}}(\mathbf{A})$, $r_{\theta}(\mathbf{A}), \sigma_{b}(\mathbf{A})$ and $r_{b}(\mathbf{A})$ are the correspondent values predicted from the adopted yield criterion. The weighting factors, $w_{\sigma_{\theta}^{T}}, w_{\sigma_{\theta}^{C}}, w_{r_{\theta}}, w_{\sigma_{b}}$ and $w_{r_{b}}$ are used to balance the influence of the experimental data. The anisotropy coefficients $C_{44}$ and $C_{55}$, of CPB06, and $c_{4}$ and $c_{5}$, for the YLD91, are associated with out-of-plane properties, difficult to evaluate for metallic sheets. Therefore, they are considered equal to the isotropic value of 1.0. In brief, the number of parameters to be identified for the CPB06 yield criterion is eight: seven anisotropy parameters and $k$. For the YLD91 yield criterion this number reduces to four, i.e. the anisotropy parameters, since $m=8$.

Both yield criteria were implemented in the in-house code DD3MAT, enabling the use of all the experimental data defined in Eq. (11) in the parameters identification procedure. The optimization algorithm implemented in DD3MAT is based on a downhill simplex method, which is a derivative free method. For the CPB06 yield criterion, the identification procedure assumes a range of $[-1,1]$ for $k$ and a range of $[-5,5]$ for all the other parameters. For the YLD91, the range considered for the anisotropy parameters is $[0,5]$.

\subsection{Application to a 2090-T3 aluminum alloy.}

The experimental data for the 2090-T3 aluminum alloy is presented in Table 1, and considers results from both uniaxial tension and compression tests, as well as for biaxial tension and disk compression [15].

The anisotropy parameters were identified for both the CPB06 and the YLD91, in order to compare their ability to accurately describe the materials' mechanical behavior. Figure 1 presents the comparison between numerical and experimental $r$-values as well the yield stress in tension (labelled "T") and compression (labelled "C"), for both yield criteria.
Table 1. Experimental data obtained from uniaxial tension, compression, biaxial tension and disk compression tests, for the 2090-T3 aluminum alloy [15].

\begin{tabular}{cccc}
\hline Test direction $\left[^{\circ}\right]$ & $r$-value & $\sigma_{\theta}^{Y_{T}}[\mathrm{MPa}]$ & $\sigma_{\theta}^{Y_{C}}[\mathrm{MPa}]$ \\
\hline 0 & 0.21 & 279.62 & 248.02 \\
15 & 0.33 & 269.72 & 260.75 \\
30 & 0.69 & 255.00 & 255.00 \\
45 & 1.58 & 226.77 & 237.75 \\
60 & 1.05 & 227.50 & 245.75 \\
75 & 0.55 & 247.20 & 263.75 \\
90 & 0.69 & 254.45 & 266.48 \\
$\sigma_{b}$ & & 289.40 & \\
$r_{b}$ & 0.670 & & \\
\hline
\end{tabular}

Regarding the $r$-values, both yield criteria seem to describe well the materials mechanical behavior, except for the test performed at $45^{\circ}$. The CPB06 yield criterion presents a better prediction, mainly between $60^{\circ}$ and $90^{\circ}$. Also, even though no experimental data is available, the numerically predicted compression $r$-values are also represented in this figure, highlighting the difference in behavior of this material in tension and compression. Note that the $r$-values in compression are higher than the ones in tension for the rolling direction until about $45^{\circ}$, at which they become lower until the transverse direction.

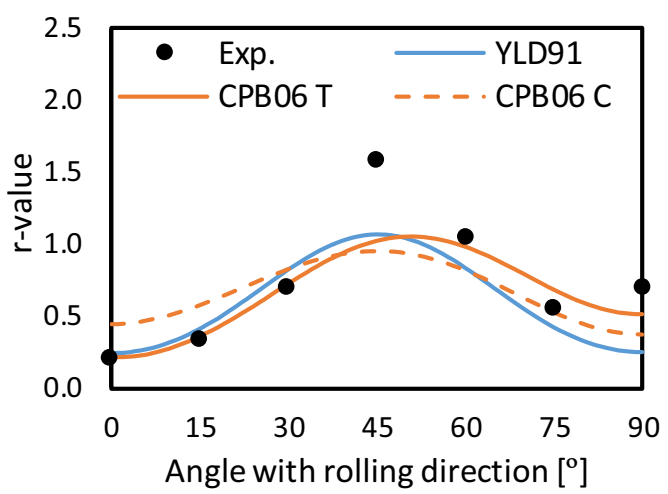

(a)

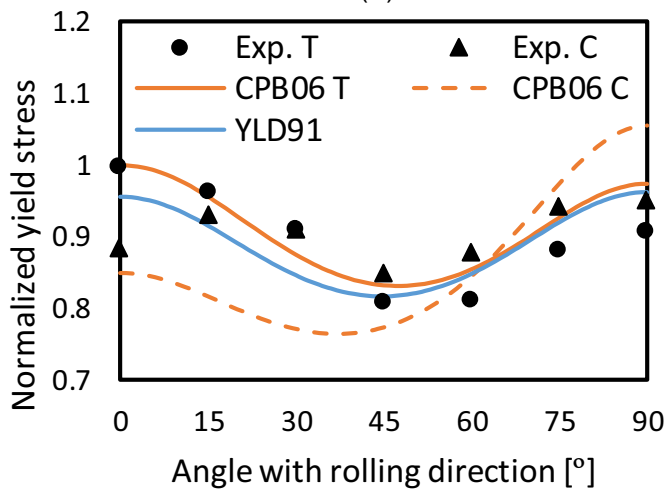

(b)

Figure 1. Experimental and predicted (a) $r$-values and (b) normalized yield stresses for the 2090-T3 aluminum alloy.

As for the yield stresses in tension both yield criteria show a similarly good agreement with the experimental results, with the CPB06 being more accurate mainly for angles closer to the rolling direction. Considering the yield stresses in compression, note that the YLD91 prediction is 
the same as for the tension case. The CPB06 seems to predict the main shape of the yield stresses evolution, even though it under predicts the values from $0^{\circ}$ to $45^{\circ}$ and over predicts from $45^{\circ}$ to $90^{\circ}$.

Figure 2 presents the normalized yield surfaces ( $Y_{0}=279.62 \mathrm{MPa}$ ) predicted, for both yield criteria, for the 2090-T3 aluminum alloy, in the plane $\sigma_{1}, \sigma_{2}$, with $\sigma_{3}=0$. The YLD91 predicted surface presents a point symmetry, implying that the behavior of the material in tension is the same as in compression. The CPB06 predicted yield surface is not symmetric, thus better representing the material behavior for different stress states. Note that the condition $Y_{0}=\sigma_{0}^{Y_{\mathrm{T}}}$ is imposed by the CPB06 yield criterion [13], while for the YLD91 this condition is not imposed in the anisotropy parameters identification procedure.

When compared with the experimental results, the CPB06 closely predicts the yield stresses both in tension and compression, thus predicting the different ratios obtained for the two principal directions shown. Table 2 shows the ratios obtained for these axes and also the one predicted along the thickness direction, corresponding to the biaxial one (with $\sigma_{3}^{\mathrm{T}} / \sigma_{3}^{\mathrm{C}}=\sigma_{b}^{\mathrm{C}} / \sigma_{b}^{\mathrm{T}}$ ). In fact, the numerically obtained ratios for the $\sigma_{1}$ and $\sigma_{2}$ axis are very close to the ones obtained experimentally. Note that the experimental ratio for the $\sigma_{3}$ is not available, thus only the numerically predicted one is shown. Regarding the biaxial values, the experimental and predicted $\sigma_{b}$ and $r_{b}$ are presented in Table 3 .

As for the $\sigma_{b}$ value, none of the yield criteria show a good agreement with the experimental value, with the YLD91 yield criterion predicting a slightly closer value. As for the $r_{b}$ value, both yield criteria over predict its value when comparing with the experimental one. Finally, Table 4 shows the anisotropy parameters obtained for both the CPB06 and YLD91 yield criteria.

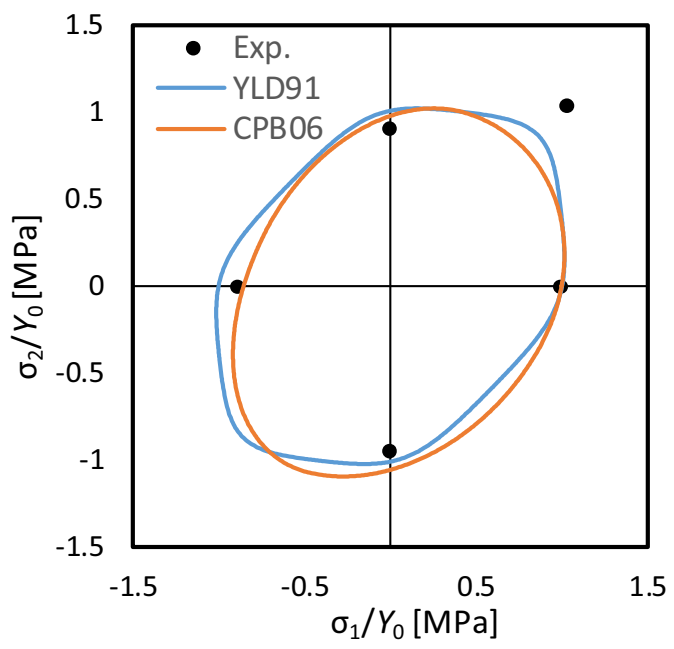

Figure 2. Predicted yield surfaces, in the $\sigma_{1}, \sigma_{2}$ plane, for the YLD91 and CPB06 yield criteria.
Table 2. Ratios obtained for the three principal axis for the 2090-T3 aluminum alloy.

\begin{tabular}{cccc}
\hline & $\left(\sigma_{1}^{\mathrm{T}} / \sigma_{1}^{\mathrm{C}}\right)$ & $\left(\sigma_{2}^{\mathrm{T}} / \sigma_{2}^{\mathrm{C}}\right)$ & $\left(\sigma_{3}^{\mathrm{T}} / \sigma_{3}^{\mathrm{C}}\right)$ \\
\hline Experimental & 1.1274 & 0.9549 & - \\
CPB06 & 1.1756 & 0.9217 & 1.0361 \\
\hline
\end{tabular}

Table 3. Experimental and numerically predicted biaxial tensile values for the 2090-T3 aluminum alloy.

\begin{tabular}{ccc}
\hline & $\sigma_{b}$ & $r_{b}$ \\
\hline Experimental & 289.40 & 0.670 \\
YLD91 & 230.83 & 0.971 \\
CPB06 & 219.42 & 0.968 \\
\hline
\end{tabular}

Table 4. Anisotropy parameters identified for both the YLD91 and CPB06 yield criteria, for the 2090-T3 aluminum alloy.

\begin{tabular}{cccc}
\hline & CPB06 & & YLD91 \\
\hline$C_{11}$ & 0.9353 & $c_{1}$ & 1.0692 \\
$C_{22}$ & 0.4171 & $c_{2}$ & 1.3002 \\
$C_{33}$ & 0.7278 & $c_{3}$ & 0.8560 \\
$C_{66}$ & 0.7758 & $c_{6}$ & 1.2132 \\
$C_{23}$ & -0.1194 & $m$ & 8 \\
$C_{13}$ & 0.0971 & & \\
$C_{12}$ & 0.1591 & & \\
$k$ & 0.0922 & & \\
\hline
\end{tabular}

\section{Cup drawing of a circular blank}

The numerical simulation of a cup drawing of a circular cup was performed in order to analyze the influence of the $\mathrm{SD}$ effect in the earing profile. This test is based on the work of Yoon et al. (2000) [10].

\subsection{Problem description}

The schematic of the cup drawing process, together with the main dimensions, is shown in Figure 3. Due to geometrical and material symmetries, only a quarter of the global structure is modelled. The contact with friction conditions is described by Coulomb's law, using a constant friction coefficient, $\mu$, of 0.1 . The blank sheet is circular in shape with a radius of $158.76 \mathrm{~mm}$ and thickness of $1.6 \mathrm{~mm}$. The blank-holder force has a value of $22.2 \mathrm{kN}$, which correspond to the minimum value predicted to avoid wrinkles [10].

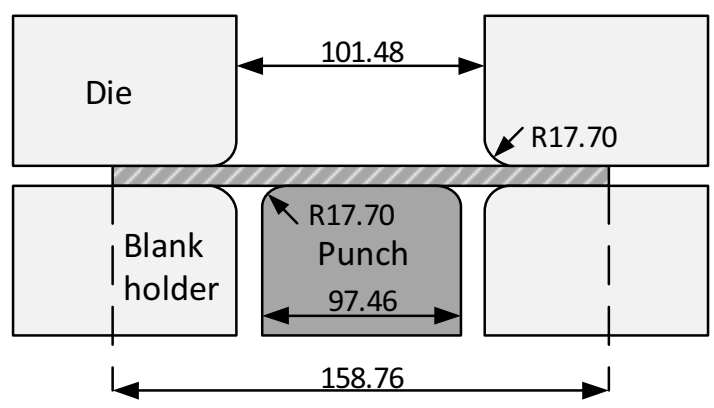

Figure 3. Schematic of the cup drawing and main dimensions. 
The sheet is discretized with 8-node hexahedral finite elements, combined with a selective reduced integration technique [16]. Figure 4 shows the in-plane sheet discretization used. Two elements were used in the thickness direction, leading to a total of 19648 elements.

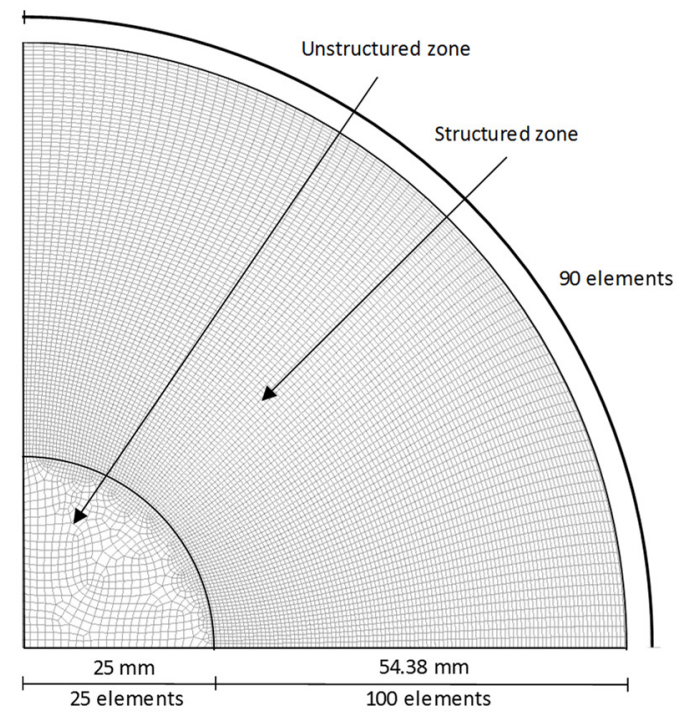

Figure 4. In-plane blank sheet discretization.

The material's mechanical behavior is assumed to be isotropic in the elastic regime, being described by the Young's modulus, $E$, and the Poisson ratio, $v$. The plastic behavior is described using an isotropic work hardening Swift type law, given by

$$
Y\left(\bar{\varepsilon}^{\mathrm{p}}\right)=K\left(\varepsilon_{0}+\bar{\varepsilon}^{\mathrm{p}}\right)^{n},
$$

where $Y$ is the flow stress and $\bar{\varepsilon}^{\mathrm{p}}$ is the equivalent plastic strain. The elastic properties and material parameters of the hardening law used for the simulation are presented in Table 5. The YLD91 and CPB06 anisotropy parameters used in the simulation are the ones presented in Table 4.

Table 5. Elastic properties and material parameters of the work hardening law.

\begin{tabular}{ll}
\hline Elastic properties & Isotropic hardening (Swift law) \\
\hline$E=74[\mathrm{GPa}]$ & $K=646[\mathrm{MPa}]$ \\
$v=0.34$ & $\varepsilon_{0}=0.025$ \\
& $n=0.227$ \\
\hline
\end{tabular}

\subsection{Results and discussion}

The numerically predicted punch force and blank holder displacement with punch displacement are presented in Figure 5. Regarding the blank holder displacement, the results indicate a higher gap with the die for the CPB06, which corresponds to a thicker flange. In order to avoid excessive ironing of the flange by the blank holder, when the displacement inverts, a limit of $0.2 \mathrm{~mm}$ is imposed.

The punch force evolution is very similar for both yield criteria throughout the process. At around $35 \mathrm{~mm}$ a pronounced decrease in the force is noticed. For the CPB06, this stepped decrease occurs sooner, indicating that the blank loses contact with the blank holder sooner in the process. This indicates that the height of the formed cup is lower, for the CPB06.

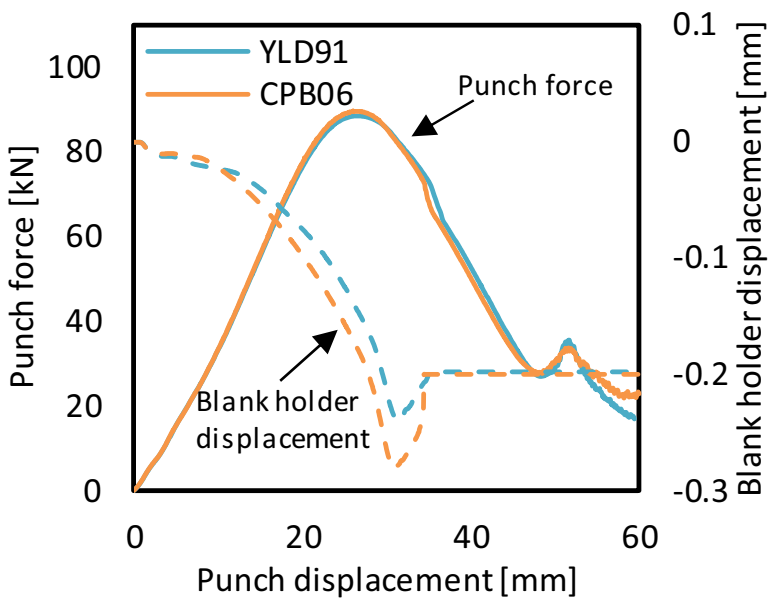

Figure 5. Numerically predicted punch force and blank holder displacement with punch displacement.

The comparison between experimental and numerically predicted cup height $v s$. angle from rolling direction, after drawing, is presented in Figure 6. The YLD91 predicts a height that is higher than the one predicted with the CPB06, between $0^{\circ}$ and $75^{\circ}$. Note that the shape of the ear for both yield criteria is nearly the same. Negligible differences can be seen between $60^{\circ}$ and $90^{\circ}$. Also, the CPB06 predicted location for the maximum height seems to correlate better with the experimental one.

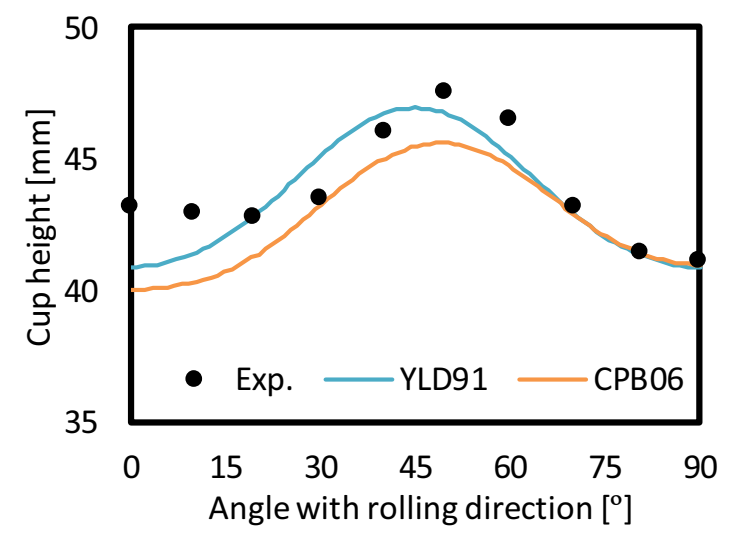

Figure 6. Comparison between experimental and numerically predicted cup height vs. angle from rolling direction.

In order to understand the differences in the predicted cup height, the stress and strain states evolutions during the cup forming process were analyzed for points located on the outermost flange, at every $15^{\circ}$ from the rolling direction. This analysis was performed assuming that, as long as the stress component in the thickness direction is small, the outer flange will be submitted to a compression stress state, as shown in Figure 7. Therefore, the material behavior will be dictated by the stress and $r$-values predicted for this stress state, which for the YLD91 are the same as for a tensile stress state. 


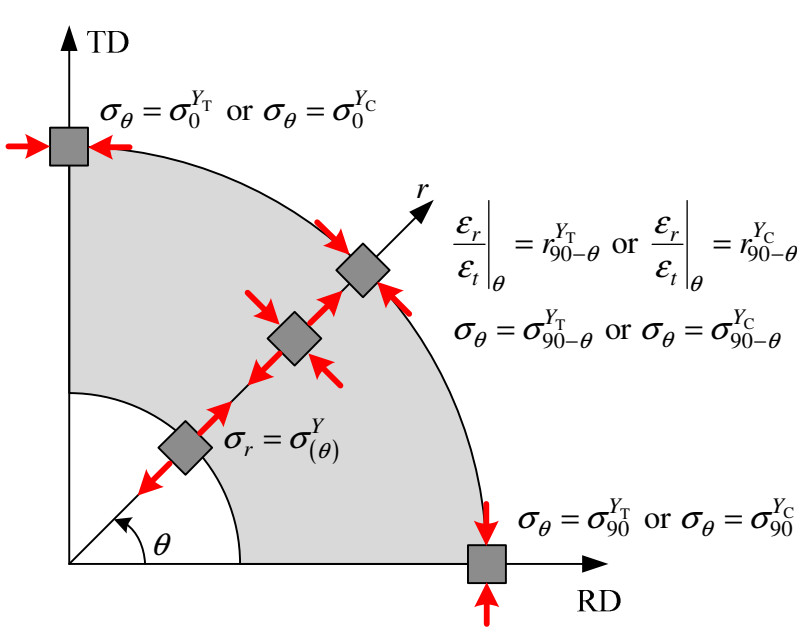

(a)

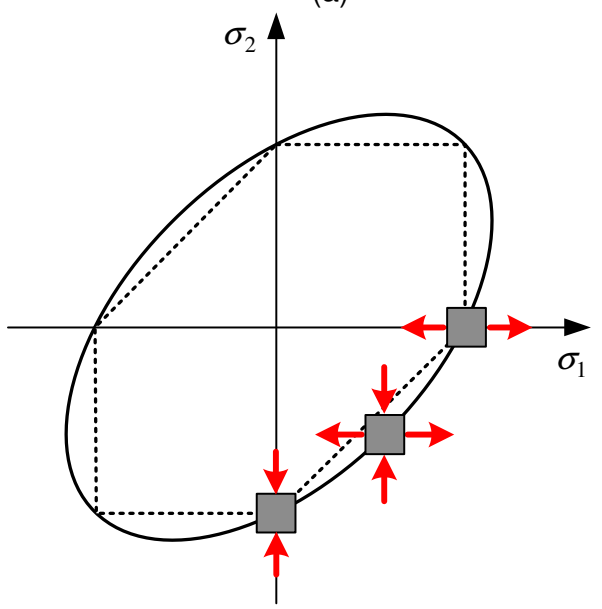

(b)

Figure 7. Deformation of an element on the flange: (a) stress states on the flange and (b) stress states on the yield surface (adapted from Yoon et al. 2011 [17]).

Figure 8 presents the evolution of the ratio between the plastic strain along the radial and the thickness direction, estimated from the numerical results, and the $r$-values estimated, for both yield criteria. The results are presented to a maximum punch stroke of $50 \mathrm{~mm}$, corresponding approximately to the instant at which the cup completely enters the die cavity. Considering the YLD91 yield criterion, the $\varepsilon_{r} / \varepsilon_{t}$ and estimated $r$-values show a good agreement, meaning that the stress state component in the thickness direction is small, thus not altering the compression stress state in the circumferential direction, for the material points located in the outer flange. Also, it is possible to observe that the yielding of the points occurs at approximately the same instant $(8 \mathrm{~mm})$. Regarding the CPB06 yield criterion, the agreement between the $\varepsilon_{r} / \varepsilon_{t}$ and estimated $r$-values (compression stress state, as shown in Figure 1 (a)) is also very good. Note that, in this case, the material points located in the outermost flange do not yield at the same instant. The point corresponding to $0^{\circ}$ yields last, for a displacement of about $11.5 \mathrm{~mm}$, followed of the point at $15^{\circ}$, for a displacement of about $8.5 \mathrm{~mm}$. This behavior is coherent with the fact that the cup height predicted by the CPB06 yield criterion is lower for angles closer to the rolling direction, i.e. since it yields later, the final cup height is lower.

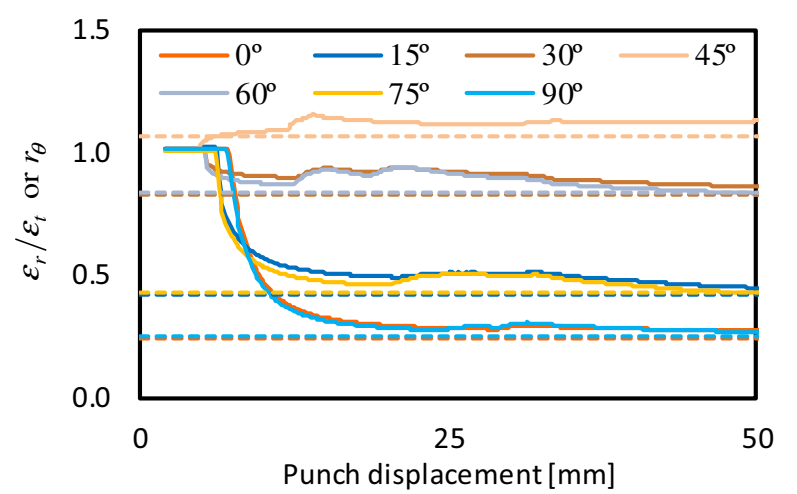

(a)

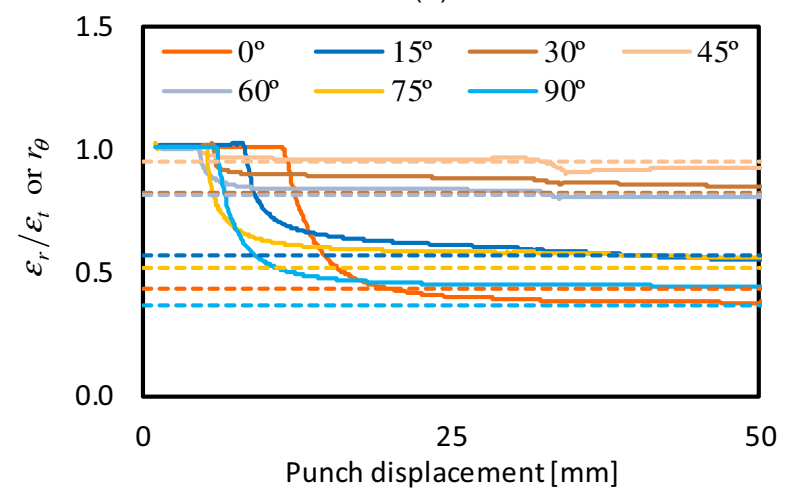

(b)

Figure 8. Evolution, with the punch displacement, of $\varepsilon_{r} / \varepsilon_{t}$ (solid lines) and $r$-values (dashed lines) estimated with (a) YLD91 and (b) CPB06 yield criteria.

Figure 9 presents the evolution of the ratio between the circumferential stress, estimated from the numerical results, and the ratio between the yield stress, estimated with both the YLD91 and CPB06 yield criteria, and $Y_{0}$. For the CPB06 yield criterion, the yield stresses considered are the ones predicted for compression (Figure 1 (b)). For both yield criteria there is a good correlation between the circumferential stress normalized by the flow stress, $\sigma_{\theta} / Y$, and the normalized yield stress values estimated with both the YLD91 and CPB06 yield criteria, $\sigma_{90-\theta} / Y_{0}$. These results are in accordance with the fact that the compression yield stresses in the rolling direction will have a direct impact on the material behavior at the transverse direction, as shown in Figure 7. In fact, the behavior of the rim in the direction defined by $\theta$ (with the rolling direction) is controlled by the material compression properties in the direction defined by $90-\theta$ [17][18]. The fact that the CPB06 compression yield stress at $90^{\circ}$ is over predicted, has as consequence the pronounced decrease in height of the earing profile, mainly between $0^{\circ}$ and $60^{\circ}$. Also, the contact with friction, in this problem, is not expected to alter the geometry of the earing profile, since the blank holder force is almost uniformly distributed in the flange [18]. 


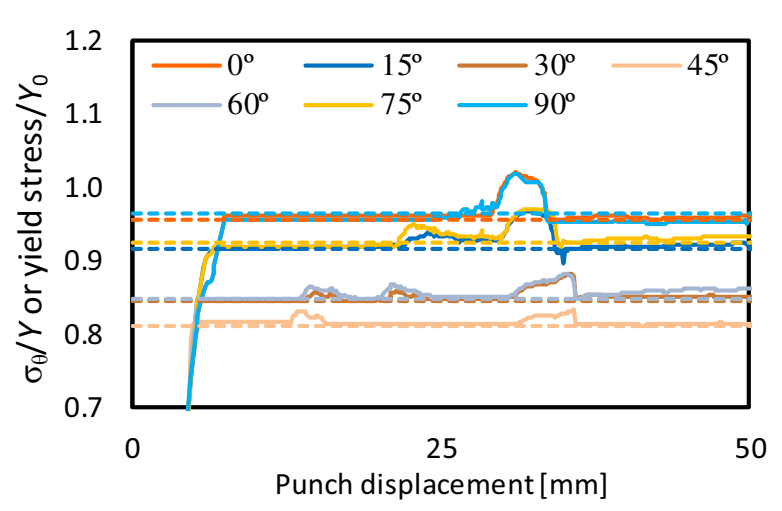

(a)

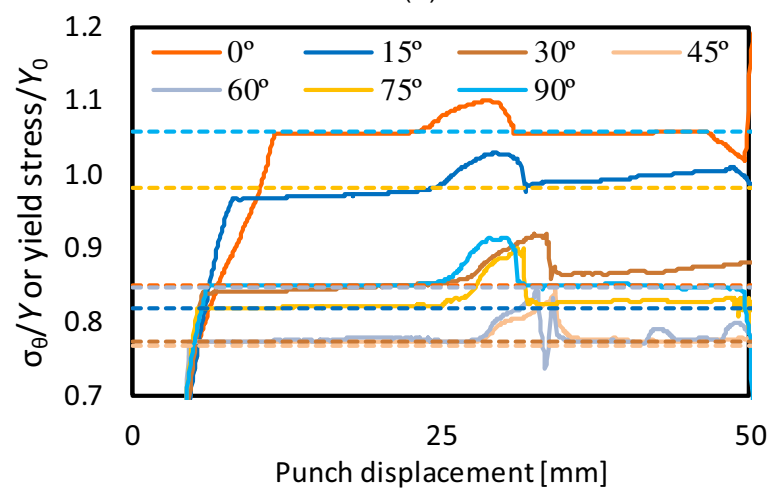

(b)

Figure 9. Evolution, with punch displacement of $\sigma_{\theta} / Y$ (solid lines) and the ratio between yield stress (dashed lines) estimated with (a) YLD91 and (b) CPB06 yield criteria and $Y_{0}$.

From Figure 9 it is possible to note that, regarding the YLD91 yield criterion, all the points of the flange yield at approximately the same time, thus being coherent with the findings from Figure 8. For the СРB06, for angles of $0^{\circ}$ and $15^{\circ}$ from the rolling direction, the material yields for a punch displacement higher than for the other directions. Again, this is coherent with the results from Figure 8, but also from Figure 1 (b). The fact that the normalized yield stress values estimated for a compression test performed at $75^{\circ}$ and $90^{\circ}$ to the rolling direction are higher than 1.0, on the contrary with the others, delays the yielding for material points located between the rolling direction and $15^{\circ}$. This also allows saying that the fact that the cups height predicted with the CPB06 is lower for points closer with the rolling direction is directly related with the later yielding of this material.

Figure 10 presents the thickness strains evolution measured experimentally and the ones predicted, for both the YLD91 and CPB06 yield criteria, for the rolling and transverse direction. Regarding the YLD91 yield criterion, the evolution in the rolling and transverse directions are the same, with no observable differences. On the other hand, the predictions for the CPB06 yield criterion show that the thickness strain in the transverse direction is always higher than the one in the rolling direction. This result is, in fact, coherent with the experimental results even thought, for both yield criteria, the strains are globally over predicted. The fact that the thickness strain in the rolling direction is lower, for the $\mathrm{CPB} 06$, also correlates with the lower cup height in this direction.

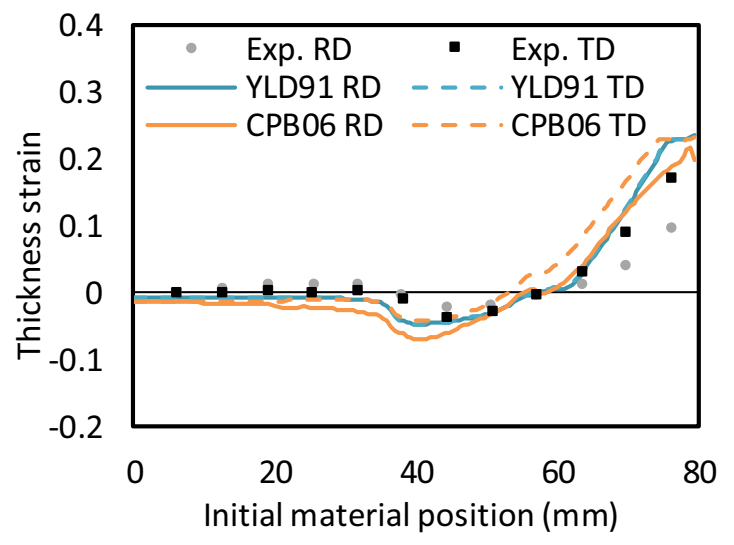

Figure 10. Evolution of the predicted and measured strain, for the YLD91 and CPB06 yield criteria, regarding the rolling and transverse directions.

\section{Conclusions}

In this work, the anisotropy parameters for the CPB06 yield criterion, which accounts for tension-compression asymmetry, as well as for the YLD91, were determined. The results show that both yield criteria can describe the yield stresses and $r$-values in tension, with the CPB06 also describing the materials' mechanical behavior for compression stress states.

The results for the numerical simulation of the cup drawing show that there is a direct influence of the yield criteria prediction of the compression yield stresses on the final shape of the cup, since this is the main stress state in the cups' flange during drawing.

Thus, a good prediction of the material directionalities, including the compression yield stresses, is important for the numerical simulation of drawing processes, even for materials exhibiting a small tension-compression asymmetry since it controls the overall accuracy of the earing profile.

\section{Acknowledgements}

The authors gratefully acknowledge the financial support of the Portuguese Foundation for Science and Technology (FCT) under projects with reference PTDC/EMSTEC/0702/2014 and PTDC/EMS-TEC/6400/2014. The first author is also grateful to the FCT for the $\mathrm{PhD}$ grant SFRH/BD/98545/2013.

\section{References}

1. O. Cazacu, B. Plunkett, F. Barlat, Int. J. Plast. 22, 1171-1194 (2006).

2. O. Cazacu, F. Barlat, Math. Mech. Solids 6, 613-630 (2001).

3. F. Barlat, H. Aretz, J.W. Yoon, M.E. Karabin, J.C. Brem, R.E. Dick, Int. J. Plast. 21, 1009-1039 (2005).

4. D. Banabic, Sheet Metal Forming Processes, Springer- 
Verlag Berlin Heidelberg, 2010.

5. J. Yoon, F. Barlat, R. Dick, M. Karabin, Int. J. Plast. 22, 174-193 (2006).

6. D.J. Lege, F. Barlat, J.C. Brem, Int. J. Mech. Sci. 31, 549-563 (1989).

7. K. Pöhlandt, D. Banabic, K. Lange, in:, ESAFORM 2002, Krakow, Poland., 2002, pp. 723-727.

8. F. Barlat, J.C. Brem, J.W. Yoon, K. Chung, R.E. Dick, D.J. Lege, F. Pourboghrat, S.H. Choi, E. Chu, Int. J. Plast. 19, 1297-1319 (2003).

9. F. Barlat, D.J. Lege, J.C. Brem, C.J. Warren, in:, T.. Lowe, A.. Rollett, P.. Follansbee, G.. Daehn (Eds.), TMS Annu. Meet., TMS, New Orleans, LA, 1991, pp. 189-203.

10. J.W. Yoon, F. Barlat, K. Chung, F. Pourboghrat, D.Y. Yang, Int. J. Plast. 16, 1075-1104 (2000).

11. M. Tritschler, a. Butz, D. Helm, G. Falkinger, J. Kiese, Int. J. Mater. Form. 7, 259-273 (2014).

12. F. Barlat, Y. Maeda, K. Chung, M. Yanagawa, J.C. Brem, Y. Hayashida, D.J. Lege, K. Matsui, S.J. Murtha, S. Hattori, R.C. Becker, S. Makosey, J. Mech. Phys. Solids 45, 1727-1763 (1997).

13. P.D. Barros, M.C. Oliveira, J.L. Alves, A. AndradeCampos, L.F. Menezes, in:, P. Hora (Ed.), Institute of Virtual Manufacturing, ETH Zurich, Zurich, 2015, pp. 149-154.

14. F. Barlat, D.J. Lege, J.C. Brem, Int. J. Plast. 7, 693712 (1991).

15. F. Moayyedian, M. Kadkhodayan, (2014) 2804-2826.

16. T.J.R. Hughes, Int. J. Numer. Methods Eng. 15, 14131418 (1980).

17. J.W. Yoon, R.E. Dick, F. Barlat, Int. J. Plast. 27. 11651184 (2011).

18. P.D. Barros, D.M. Neto, J.L. Alves, M.C. Oliveira, L.F. Menezes, Rom. J. Tech. Sci. - Appl. Mech. 60, 105-136 (2015). 